P. Leiria-Pinto ${ }^{1,2}$, J. Marques ${ }^{1,2}$, E. Finelli ${ }^{1}$, C. Alves ${ }^{1}$, M. Alves ${ }^{3,4}$, D. Trincão ${ }^{1}$, N. Pinto ${ }^{1}$, P. Carreiro-Martins ${ }^{1,2}$, A. L. Papoila ${ }^{3,4}$, N. Neuparth ${ }^{1,2}$

\title{
Cross-cultural validation of the Portuguese from Portugal version of the Test for Respiratory and Asthma Control in Kids (TRACK) questionnaire
}

\author{
${ }^{1}$ Department of Immunoallergology, Hospital Dona Estefânia, Centro Hospitalar Universitário de Lisboa Central \\ (CHULC), EPE, Lisbon, Portugal \\ ${ }^{2}$ Comprehensive Health Research Center/Chronic Diseases Research Center (CHRC/CEDOC), Integrated Pathophysiolo- \\ gical Mechanisms Research Group, Nova Medical School, Lisbon, Portugal \\ ${ }^{3}$ Research Centre, CHULC, EPE, Lisbon, Portugal \\ ${ }^{4}$ Centre of Statistics and its Applications (CEAUL), Lisbon, Portugal
}

\section{KEY WORDS}

TRACK; questionnaire; asthma control; preschool; validation.

\section{Corresponding author}

Paula Leiria Pinto

Department of Immunoallergology

Hospital Dona Estefânia

CHULC

Rua Jacinta Marto

1169-045 Lisboa, Portugal

ORCID ID: 0000-0001-9027-8388

E-mail: pleiriapinto@gmail.com

Doi

10.23822/EurAnnACI.1764-1489.174

\begin{abstract}
Summary
Introduction. The Test for Respiratory and Asthma Control in Kids (TRACK) is a tool to assess asthma control in preschool children. This study aims to validate the Portuguese from Portugal version of the TRACK questionnaire. Methods. A prospective cohort study was carried out to assess their psychometric characteristics. Caregivers of 141 children under age 5 with asthma symptoms were enrolled. Results. Internal reliability was close to 0.70 (Cronbach's $\alpha$ ). The test-retest reliability was 0.87. TRACK scores were different between well, partially, and non-controlled asthma groups $(p<0.001)$. Patients rated as having better control showed an increase in TRACK scores. Conclusions. The Portuguese version of the TRACK questionnaire is accurate and reliable for monitoring asthma control. Its use may help to overcome challenges with the management of this age group.
\end{abstract}

\section{Impact statement \\ This version of the TRACK questionnaire showed to be valid for use in Portugal as an accurate and reliable tool to monitor asthma control in preschool children.}

\section{Introduction}

Asthma is a common chronic disease affecting around $6.5 \%$ of preschool children in Portugal (1), and accounts for high morbidity and costs, particularly when it becomes uncontrolled (2). Moreover, young children are more prone to develop exacerbations and have less favourable responses to asthma treatments $(3,4)$ than older children.
According to current guidelines, the goal of the treatment is to achieve and maintain asthma control (5). Moreover, Global INitiative for Asthma (GINA) criteria have been accepted as the standard for physicians assessing asthma control. Questionnaires provide a standardized evaluation of asthma control perception and its use in clinical practice may easily help clinicians to identify uncontrolled preschool asthmatic children (6). 
The Test for Respiratory and Asthma Control in Kids (TRACK) is a validated questionnaire that determines respiratory control in children younger than five years of age with symptoms consistent with asthma (7-10). Furthermore, TRACK encompasses both impairment and risk domains assessment, unlike most of the childhood asthma control questionnaires that only capture parent-reported impairment (7).

This questionnaire was already translated and validated into other languages (11-13), including Brazilian Portuguese (14), supporting a wider use across the world. Considering the recognized cultural-linguistic differences between Portuguese from Brazil and Portugal, this study aimed to translate and validate the Portuguese from Portugal version of the TRACK questionnaire.

\section{Materials and methods}

\section{Study design}

An observational, prospective cohort study was conducted at the Allergy and Immunology Department of Dona Estefânia Hospital (Lisbon, Portugal). The study comprised two visits with an interval of two to six weeks, during the period September 2017 through June 2018, and took into account the COSMIN checklist (15).

\section{Study population}

Children younger than five years old, with Portuguese-speaking parents, and with a medical diagnosis of asthma or with symptoms consistent with asthma, were eligible after their parents' consent. Inclusion criteria were either a history of having two or more episodes of wheezing, shortness of breath or cough that lasted more than 24 hours, or improvement of respiratory symptoms with the use of an aerosolized bronchodilator. Children with respiratory diseases other than asthma or with other chronic disorders or congenital abnormalities were excluded from the study.

\section{TRACK Questionnaire}

The TRACK is a five-item caregiver-completed questionnaire that assesses asthma control in children under the age of five. The first four items evaluate impairment based on the frequency of respiratory symptoms, waking up at night because of these symptoms, activity limitation in the last four weeks, and frequency of rescue medication use in the last three months. The fifth item addresses the risk domain, such as the frequency of oral corticosteroid (OCS) use over the past year.

Items may be scored between 0 to 20 , and the total score for the whole questionnaire ranges from 0 to 100 points. TRACK higher scores reflecting probably better asthma control and scores lower than 80 suggesting possibly non-controlled asthma (8). There is also evidence supporting that changes in TRACK scores of 10 or more points are representative of the minimal clinically important difference (MCID) (10).

This questionnaire is a trademark of AstraZeneca. To perform the present study, this company granted us permission to translate and use the TRACK questionnaire. They also agreed in cooperating in all stages of the process of adaptation, ensuring an accurate translation for Portuguese from Portugal.

\section{Cultural adaptation of the TRACK questionnaire}

The TRACK was adapted to a Portuguese context according to one of the forward-backward-translation methodologies (15, 16). This included four steps: 1) forward translation: two independent native Portuguese speakers, both fluent in English, performed the translation; 2) reconciliation of the forward translations into one preliminary translation was developed by two independent immunoallergists both fluent in English and Portuguese; 3) backward translation of the reconciled version into English was performed by two fluent in English independent translators, and an independent supervisor comparing the text with the original questionnaire for conceptual equivalence; 4) cognitive debriefing was conducted in 10 patients' caregivers representing the study population. Any uncertainties were discussed with the physicians through face-to-face interviews.

\section{Data collection}

Data regarding socio-demographic (age, children gender, and education of the mother and father) and clinical characteristics were recorded.

At baseline (V1) and follow-up (V2) visits, caregivers completed the Portuguese version of the TRACK. Each caregiver and physician rated the disease control status of every child in the previous four weeks on a visual analog scale (VAS) of 0 to 10 $\mathrm{cm}$ (completely controlled to not controlled at all) (17). Physicians also assessed asthma control level as controlled, partially controlled, or poorly controlled, based on the GINA guideline recommendations (5), and recorded the number of asthma exacerbations that needed a course of OCS, emergency visits, or hospitalizations in the last year.

Based on the presence or absence of asthma-like symptoms (episodes of wheezing, shortness of breath, or coughing) in the previous four weeks and last 12 months, the timing of respiratory symptoms was defined as currently symptomatic (episodes in the past four weeks), symptomatic in the recent past (episodes in the past 12 months but not within the past four weeks), or asymptomatic (without symptoms for more than 12 months). Therapeutic adjustments were performed at V2 and registered according to the three categories of possible decisions: step-up, no change, or step-down of the therapy level.

The local ethics committee of Centro Hospitalar Universitário de Lisboa Central approved the study. Written informed consent was obtained from each caregiver. 


\section{Statistical analysis}

To calculate the required number of subjects for this study where reliability is measured, a kappa for the null hypothesis of 0.5 , a kappa for the alternative hypothesis of 0.7 , a statistical significance level of 0.05 (two-sided test), and a power of $80 \%$ were considered. A sample size of 124 patients was obtained (18). Categorical variables were described as absolute frequencies and percentages. Continuous variables were presented as median and inter-quartile range (IQR: $\mathrm{P}_{25}-\mathrm{P}_{75}$ ).

Internal consistency of the TRACK was assessed by estimating Cronbach's alpha coefficient with the responses given by all the parents/caregivers at baseline and follow-up visits. A Cronbach's alpha coefficient estimate $\geq 0.7$ was considered acceptable.

The test-retest reliability of the TRACK was evaluated through the intra-class correlation coefficient (ICC) and Lin's concordance correlation coefficient. Additionally, Bland and Altman plot of the TRACK scores between visits was constructed. For this analysis, only symptomatic in the recent past or asymptomatic patients that remained clinically stable in the follow-up visit 2 to 4 weeks later, were considered.

The criterion validity was assessed by comparing TRACK scores among patient subsets differing on the level of the GINA asthma control (controlled, partially controlled, and non-controlled asthma). Additionally, the screening accuracy of TRACK was evaluated using the receiver operating characteristic (ROC) curve, assuming the hypothesis that higher scores increased the odds of having controlled asthma. It was also calculated the percentage of correctly classified, using 80 as the TRACK cut-off point.

The construct validity was studied by comparing TRACK scores across the three categories of the timing of respiratory symptoms (currently symptomatic, symptomatic in the recent past, and asymptomatic), and with the therapeutic decision.

Still, regarding construct validity, the discriminative properties of the TRACK were tested by classifying patients into different groups based on the VAS asthma score of caregivers and physicians. As there is no consensus about anchor points that should be used to classify the levels of asthma control, we decided to attribute equal intervals. Thus, patients with a VAS score $<3.3$ were considered as having their asthma controlled, patients with a VAS score between 3.3 and 6.6 as partially controlled asthma, and patients with a VAS score $>6.6$ as non-controlled asthma. TRACK's median score of all groups was compared with the Kruskal-Wallis test.

The responsiveness was measured by comparing the median TRACK scores of those patients that changed between the visits in their GINA scores from partially or non-controlled asthma (GINA score $\geq 1$ ) to controlled asthma (GINA score $=0$ ). Spearman's correlation coefficients between absolute change scores (V2-V1) of TRACK and both VAS asthma scores of caregivers and physicians, were also estimated (15).
Table I - Characteristics of patients at baseline.

\begin{tabular}{|c|c|}
\hline & Total $(n=141)$ \\
\hline Male, n (\%) & $81(57.4)$ \\
\hline Mean Age, years (SD) & $3.67(1.15)$ \\
\hline \multicolumn{2}{|l|}{ Grouped age, n (\%) } \\
\hline $0-2$ & $36(25.5)$ \\
\hline $3-5$ & $105(74.5)$ \\
\hline \multicolumn{2}{|l|}{ Caregiver education ${ }^{*}, \mathrm{n}(\%)$} \\
\hline High school not completed & $26(18.4)$ \\
\hline High school graduate & $37(26.2)$ \\
\hline Undergraduate or graduate degree & $60(42.6)$ \\
\hline \multicolumn{2}{|c|}{ Caregiver rated symptoms status, n (\%) } \\
\hline Symptomatic last 4 weeks & $87(61.7)$ \\
\hline Asymptomatic recent & $22(15.6)$ \\
\hline Asymptomatic > 1 year & $32(22.7)$ \\
\hline \multicolumn{2}{|l|}{ Caregiver rated control status $^{* *}, \mathrm{n}(\%)$} \\
\hline Controlled & $88(62.4)$ \\
\hline Partially controlled & $35(24.8)$ \\
\hline Non-controlled & $18(12.8)$ \\
\hline \multicolumn{2}{|l|}{ GINA Asthma Control, n (\%) } \\
\hline Controlled & $54(38.3)$ \\
\hline Partially controlled & $48(34.0)$ \\
\hline Non-controlled & $39(27.7)$ \\
\hline \multicolumn{2}{|l|}{ Asthma exacerbations, n (\%) } \\
\hline Courses OCS $(\geq 1)$ & $80(56.7)$ \\
\hline Emergency visits $(\geq 1)$ & $86(61.0)$ \\
\hline Hospitalization $(\geq 1)$ & $9(6.4)$ \\
\hline
\end{tabular}

*Percentages total less than 100 because some caregivers did not answer all the questions; ${ }^{* *}$ using a visual analog scale.

\section{Results}

\section{Patients sample}

We included 141 of the 148 eligible children. Dropouts were due to personal reasons (two children) and loss to follow-up (five children). The socio-demographic and clinical characteristics are described in table $\mathbf{I}$.

The median (IQR) of the TRACK scores of the 141 patients included in the study was 75.0 (65.0-90.0) and 80.0 (65.0-90.0) points at baseline and follow-up visits, respectively.

Regarding asthma control assessment, caregivers overrated controlled asthma in comparison with physicians that used GINA criteria (i.e., $62 \%$ vs $38 \%$ ). 
Figure 1 - Portuguese version of the Test for Respiratory and Asthma Control in Kids (TRACK). TRACK is a trademark of the AstraZeneca group of companies. ${ }^{\odot} 2009$ AstraZeneca LP. All rights reserved $2786505 / 09$.

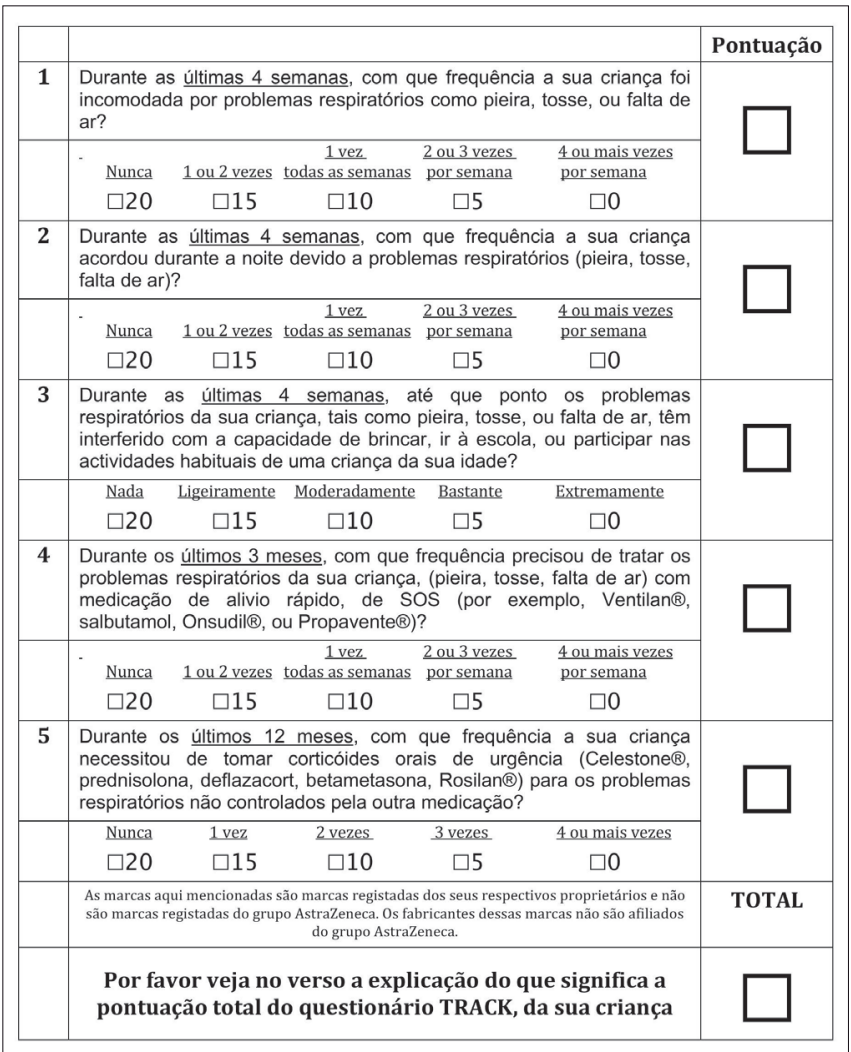

\section{Cross-cultural adaptation}

A final version for the Portuguese population was completed with few adjustments to the questions, adding "SOS" to the quick-relief medication ( $4^{\text {th }}$ question) and "emergency" for oral corticosteroids ( $5^{\text {th }}$ question), and the name of the drugs in both questions was also changed for the most used in our country (figure 1).

\section{Internal consistency}

Cronbach's alpha for the total TRACK kids' questionnaire was 0.79 (V1) and 0.69 (V2). The deletion of each item would decrease this coefficient except for the last one that would increase Cronbach's alpha to 0.81 and 0.73 , respectively.

\section{Test-retest reliability}

The ICC coefficient estimate between visits was 0.76 (95\% CI: $0.42-0.90)$ for stable patients $(\mathrm{p}<0.001)$. The median (IQR) of the TRACK scores at baseline and in the follow-up visits was not significantly different (95.0 (90.0-95.0) vs 95.0 (90.0-
Figure 2 - Agreement of TRACK scores between visits in stable patients. Dots' diameter is proportional to the number of observations with the same plot coordinates.

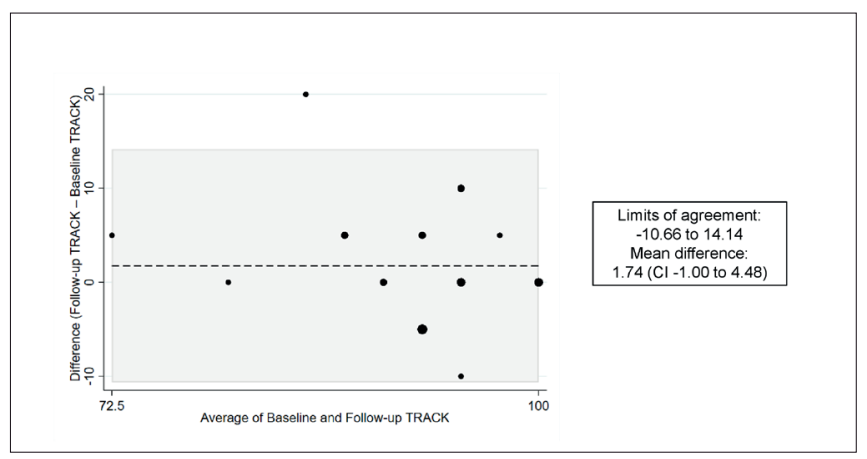

$100.0), p=0.225)$. Lin's concordance correlation coefficient was 0.56 (95\% CI: 0.28-0.84). The Bland and Altman plot shows the agreement of TRACK scores between baseline and follow-up visits (figure 2).

\section{Criterion validity}

TRACK scores were significantly different among patients with controlled asthma, patients with partially controlled asthma, and patients with non-controlled asthma (table II and figure $3 \mathbf{a}, \mathbf{b}$ ). Additionally, the TRACK questionnaire's ability to discriminate between controlled and non-controlled asthma patients at V1 was assessed through the area under the receiver operating characteristic (AUC) curve. An area of 0.90 (95\% CI: 0.85-0.95) was obtained, showing an excellent discriminative performance of the TRACK questionnaire. Using TRACK scores established control cut-off ( $<80$ versus $\geq 80$ ), control status was correctly classified in $80.1 \%$ at baseline. For V2, an AUC of 0.87 (95\% CI: 0.82-0.93) and control status was correctly classified in $78.7 \%$ of the patients.

\section{Construct validity}

TRACK scores were significantly different between patients classified as currently symptomatic, symptomatic in the recent past, and asymptomatic (70.0 (50.0-80.0) vs 85.0 (78.8-95.0) vs 95.0 (90.0-95.0) at baseline, and (70.0 (51.3-80.0) vs 85.0 (78.890.0) vs 95.0 (90.0-100.0) at the follow-up), both $\mathrm{p}<0.001$. Moreover, asymptomatic patients had the TRACK highest values while currently symptomatic children had the lowest ones. Based on VAS scores, the median (IQR) of the TRACK scores at baseline between children classified by parents and physicians as having controlled $v$ s partially or non-controlled asthma were, most of them, also significantly different (table III).

Comparing the TRACK scores among a decision of "step-up", "no change" and "step-down" therapy groups, significantly higher scores were found along with the reduction of the therapy (table IV). 
Table II - TRACK scores by GINA levels of asthma control at baseline and follow-up.

\begin{tabular}{|c|c|c|c|c|}
\hline & \multicolumn{4}{|c|}{ Control rating - GINA criteria } \\
\hline & Controlled & Partially controlled & Non-controlled & p value \\
\hline TRACK scores V1 & $90.0(85.0-95.0)$ & $75.0(65.0-80.0)$ & $55.0(40.0-70.0)$ & $<0.001$ \\
\hline TRACK scores V2 & $90.0(85.0-95.0)$ & $75.0(67.5-85.0)$ & $55.0(45.0-70.0)$ & $<0.001$ \\
\hline Median (P25-P75) n (\%) & $69(48.9)$ & $37(26.2)$ & $35(24.8)$ & \\
\hline
\end{tabular}

\section{Responsiveness}

The median TRACK scores at baseline of partially and non-controlled asthmatic children were significantly lower than those obtained in the follow-up visit when asthma became controlled (67.5 (45.0-75.0) vs 85.0 (70.0-86.3), $\mathrm{p}<0.001)$.

Spearman's correlation coefficients' estimates of TRACK change scores with caregivers and physicians VAS asthma change scores, between follow-up and baseline visits, showed moderate to strong associations, both $\mathrm{p}<0.001$ (table V).

\section{Discussion}

This study provides evidence that Portuguese from Portugal version of the TRACK is a reliable and valid tool for assessing respiratory control problems, as demonstrated by their adequate psychometric properties, comparable to those of the original validation (8).

The internal consistency, close to 0.70 , increasing when deleting the fifth item from the scale, was considered acceptable. Similar results were reported by other authors $(8,9)$ because this last item reflects another different dimension of control, the risk instead of impairment. These related two domains may change independently (19), allowing us to accept values lower than the desirable, in the range of 0.60 to 0.69 .
Despite the interval of 2-4 weeks between the test-retest, the reliability found was nearly moderate to good (20) for stable patients. Looking at the confidence interval of ICC, this was quite broad, although following the results obtained either on the original (9) as in other languages validation of the TRACK (1114). Furthermore, common fluctuations of asthma symptoms in preschool children probably were contributing to these findings. Albeit there was no consensus, the GINA criteria were selected as the standard for rating asthma control levels because they are well accepted in clinical and research settings $(5-7,10)$.

Concerning criterion and construct validities, our results were similar to those of the TRACK original validation study (8, 9), with median scores of the TRACK differing significantly in the expected direction for levels of respiratory control based on GINA guidelines, the timing of respiratory symptoms, VAS asthma scores, and recommendations of changes in therapy.

Regarding discriminative accuracy, the TRACK showed also good areas under the ROC curve relative to the ratings of asthma control. The AUC values of 0.90 and 0.87 were similar or even better than those reported for the development and validation samples (8). Likewise, the questionnaire correctly classified respiratory control levels in nearly $80 \%$ of children (8).

Figure 3 - (a) TRACK scores by GINA levels of asthma control at baseline. (b) TRACK scores by GINA levels of asthma control at follow-up.

(a)

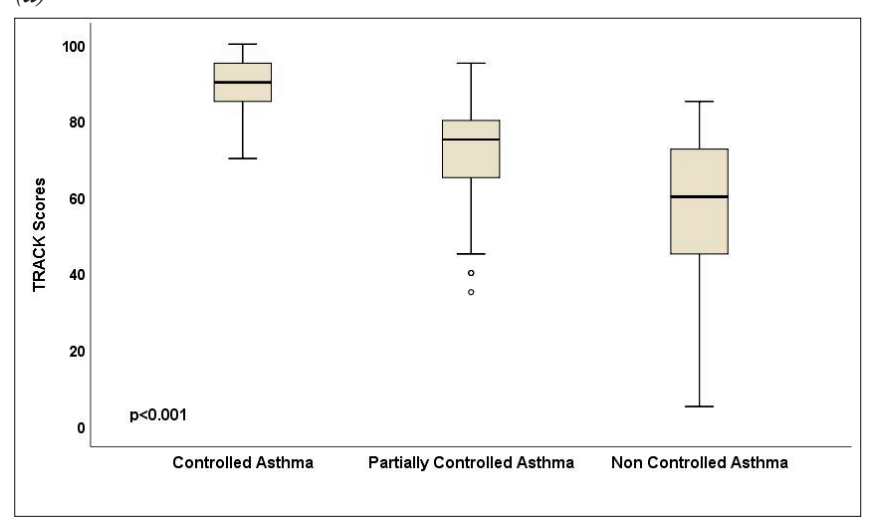

(b)

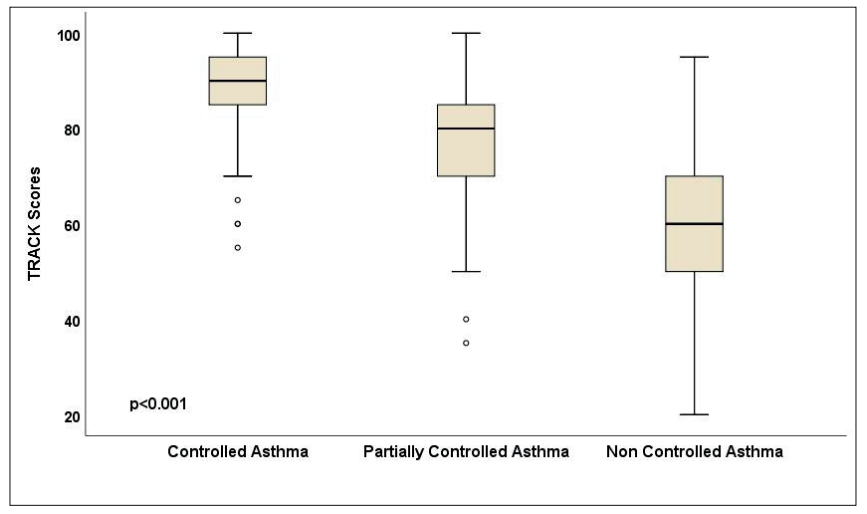


Table III - Evaluation of the discriminative ability of TRACK using asthma VAS scores classification.

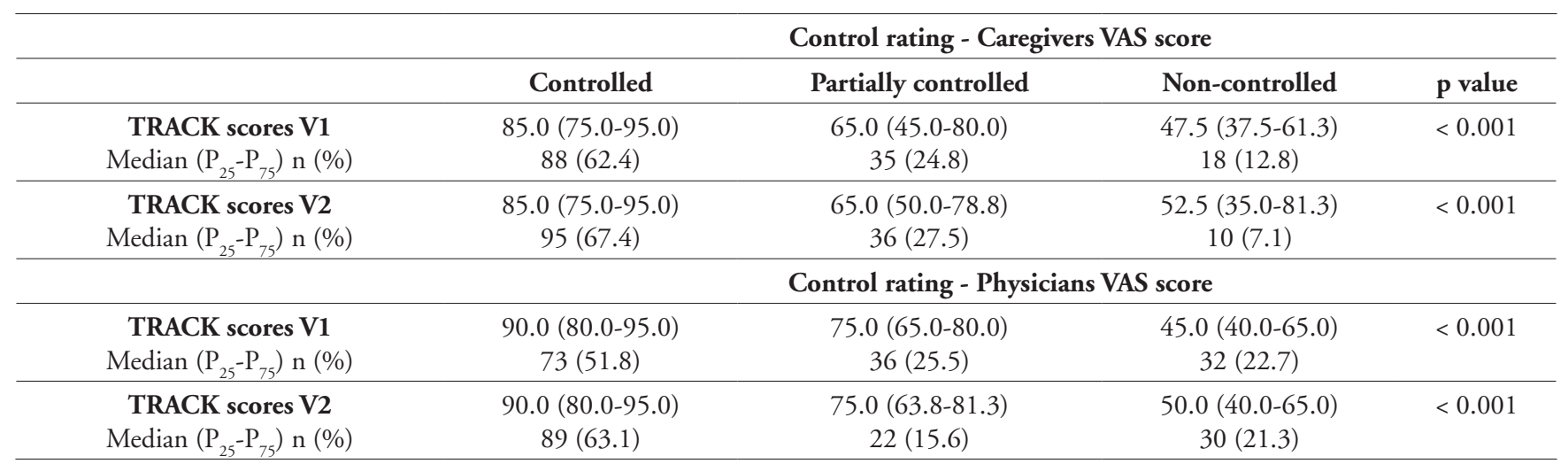

Table IV - Evaluation of the discriminative ability of TRACK using a therapeutic decision.

\begin{tabular}{cccc}
\hline & \multicolumn{3}{c}{ Therapeutic recommendation } \\
\hline TRACK scores V2 & Step-up & No change & Step-down \\
\hline Median $\left(\mathrm{P}_{25}-\mathrm{P}_{75}\right) \mathrm{n}(\%)$ & $60.0(50.0-75.0)$ & $85.0(80.0-90.0)$ & $100.0(91.3-100.0)$ \\
\hline
\end{tabular}

Good responsiveness to change in asthma control was shown when we compared median TRACK change scores among the children, grouped by the physicians' GINA, and corresponding VAS change scores. The results of this comparison suggested a parallel change of TRACK with control status, supporting the discriminant validity of the TRACK scores. Our results were also in agreement with those obtained by Wandalsen et al., in what concerns the discriminatory capacity as the reliability of the Brazilian version of TRACK (14).

Based on these findings, the implementation of the TRACK questionnaire as a tool for assessing asthma control in preschool children may improve asthma management, identifying uncontrolled patients easier. These patients require medication adjustments and consequently, their better identification may lead to significant reductions of the disease burden.

The main limitation of this study was the inclusion of a relatively small number of patients from a single center. Nevertheless, this Portuguese version of the TRACK probably exhibits similar psychometric properties in other clinical settings. Additional studies and more widespread use of TRACK will confirm this expectation. One of the main strengths of our study is the inclusion in the assessment of the asthma control of other validated tools such as
Table $\boldsymbol{V}$ - Correlation coefficients' estimates of TRACK with caregivers and physicians VAS change scores between visits (V2-V1).

\begin{tabular}{ccc}
\hline Change of scores & $\begin{array}{c}\text { Caregivers } \\
\text { VAS* asthma }\end{array}$ & $\begin{array}{c}\text { Physicians } \\
\text { VAS }^{*} \text { asthma }\end{array}$ \\
\hline TRACK & -0.545 & -0.684 \\
& $(-0.656--0.410)$ & $(-0.768--0.578)$ \\
\hline
\end{tabular}

*VAS: Visual Analog Scale.

VAS scores in addition to the GINA criteria, which overcomes any possible drawback of the selected standard.

\section{Conclusions}

In conclusion, the TRACK is a reliable and valid tool to assess asthma control in Portuguese preschool children. The findings of this study are important because asthma is highly prevalent, and the use of the Portuguese version of the TRACK may help clinicians to overcome some difficulties associated with pediatric asthma care. 


\section{Acknowledgments}

The authors express their gratitude to the participant children, their parents, and caregivers for their essential contribution. The authors thank Daniel Virella MD MSc for the methodological support.

\section{Conflict of interests}

The authors declare that they have no conflict of interests.

\section{References}

1. Ferreira-Magalhães M, Sá-Sousa A, Morais-Almeida M, et al. Asthma-like symptoms, diagnostic tests, and asthma medication use in children and adolescents: A population-based nationwide survey. J Asthma 2016;53(3):269-76.

2. Ferreira de Magalhães M, Amaral R, Pereira AM, et al. Cost of asthma in children: A nationwide, population-based, cost-of-illness study. Pediatr Allergy Immunol 2017;28(7):683-91.

3. Mahut B, Trinquart L, Delclaux C. Influence of age on the risk of severe exacerbation and asthma control in childhood. J Asthma 2011;48(1):65-8.

4. Fitzpatrick AM, Bacharier LB, Guilbert TW, et al. Phenotypes of Recurrent Wheezing in Preschool Children: Identification by Latent Class Analysis and Utility in Prediction of Future Exacerbation. J Allergy Clin Immunol Pract 2019;7(3):915-24.e7.

5. Global Initiative for Asthma: Global strategy for asthma management and prevention 2020. Available at: www.ginasthma.org. Last access date: 10/13/2020.

6. Voorend-Van Bergen S, Vaessen-Verberne AA, De Jongste JC, Pijnenburg MW. Asthma control questionnaires in the management of asthma in children: A review. Pediatr Pulmonol 2015;50(2):202-8.

7. Dinakar C, Chipps BE, Matsui EC, et al. Clinical tools to assess asthma control in children. Pediatrics 2017;139(1):e20163438.

8. Murphy KR, Zeiger RS, Kosinski M, et al. Test for Respiratory and Asthma Control in Kids (TRACK): A caregiver-completed questionnaire for preschool-aged children. J Allergy Clin Immunol 2009;123(4):833-9.e9.
9. Chipps B, Zeiger RS, Murphy K, et al. Longitudinal Validation of the Test for Respiratory and Asthma Control in Kids in Pediatric Practices. Pediatrics 2011;127(3):e737-47.

10. Zeiger RS, Mellon M, Chipps B, et al. Test for Respiratory and Asthma Control in Kids (TRACK): Clinically meaningful changes in score. J Allergy Clin Immunol 2011;128(5):983-8.

11. Buyuktiryaki B, Sahiner UM, Yavuz ST, et al. Validation of the Turkish version of "test for Respiratory and Asthma Control in Kids (TRACK)” questionnaire. J Asthma 2013;50(10):1096-101.

12. Rodríguez-Martínez CE, Nino G, Castro-Rodriguez JA. Validation of the Spanish Version of the Test for Respiratory and Asthma Control in Kids (TRACK) in a Population of Hispanic Preschoolers. J Allergy Clin Immunol Pract 2014;2(3):326-331.e3.

13. Zhang J, Zhao LX, Zhao D, et al. Reliability and validity of the Chinese version of the Test for Respiratory and Asthma Control in Kids (TRACK) in preschool children with asthma: A prospective validation study. BMJ Open 2019;9(8):e025378.

14. Wandalsen GF, Dias RG, Chong-Neto HJ, et al. Test for Respiratory and Asthma Control in Kids (TRACK): Validation of the Portuguese version. World Allergy Organ J 2018;11(1):1-7.

15. Mokkink LB, Terwee CB, Patrick DL, et al. The COSMIN checklist for assessing the methodological quality of studies on measurement properties of health status measurement instruments: An international Delphi study. Qual Life Res 2010;19(4):539-49.

16. Epstein J, Santo RM, Guillemin F. A review of guidelines for cross-cultural adaptation of questionnaires could not bring out a consensus. J Clin Epidemiol 2015;68(4):435-41.

17. Ohta K, Jean Bousquet P, Akiyama K, et al. Visual analog scale as a predictor of GINA-defined asthma control. The SACRA study in Japan. J Asthma 2013;50(5):514-21.

18. Cantor AB. Sample-size calculations for Cohen's kappa. Psychol Methods 1996;1(2):150-3.

19. Hamelmann E, von Mutius E, Bush A, Szefler SJ. Addressing the risk domain in the long-term management of pediatric asthma. Pediatr Allergy Immunol 2020;31:233-42.

20. Koo TK, Li MY. A Guideline of Selecting and Reporting Intraclass Correlation Coefficients for Reliability Research. J Chiropr Med 2016;15(2):155-63. 